\title{
Tomorrow today: organ-on-a-chip advances towards clinically relevant pharmaceutical and medical in vitro models
}

\author{
Mario Rothbauer ${ }^{a}$, Julie M. Rosser ${ }^{a}$, Helene Zirath $^{a}$, and Peter Ertl ${ }^{a, *}$ \\ *corresponding author: peter.ertl@tuwien.ac.at \\ ${ }^{a}$ Vienna University of Technology, Faculty of Technical Chemistry, Institute of Applied \\ Synthetic Chemistry, Institute of Chemical Technologies and Analytics, Getreidemarkt \\ 9/163-164, 1060 Vienna, Austria
}

all authors contributed equally

\begin{abstract}
Organ-on-a-chip technology offers the potential to recapitulate human physiology by keeping human cells in a precisely controlled and artificial tissue-like microenvironment. The current and potential advantages of organ-on-chips over conventional cell cultures systems and animal models have captured the attention of scientists, clinicians and policymakers as well as advocacy groups in the past few years. Recent advances in tissue engineering and stem cell research are also aiding the development of clinically relevant chip-based organ and diseases models with organ level physiology for drug screening, biomedical research and personalized medicine. Here, the latest advances in organ-on-a-chip technology are reviewed and future clinical applications discussed.
\end{abstract}

Keywords: Enabling technology, Organ-on-a-chip, Disease-on-a-chip, Personalized medicine, Pharmaceutical screening, in vitro - in vivo gap 


\section{Introduction}

Despite initial successes in in vivo and in vitro testing during drug trials, drug failure remains high and is accompanied by major losses in research investments. This means that, despite emerging combinatorial biochemical approaches and therapeutic strategies, regulatory approval rates for new drugs and therapies are declining. Established in vivo animal and in vitro models often do not reliably recreate the physiology of the target organ, thus poorly predicting therapeutic outcomes and possible side effects during clinical trials. To overcome these limitations, major resources have been devoted to improving experimental disease predictors to minimize patient risk and development costs. One promising strategy is chipbased organ and disease modelling. These so-called organ-on-a-chip combine recent microfluidic advances with complex three-dimensional cell biology that provide organ-like physiology and pathophysiological cellular and tissue level responses [1-3]. The inherent design flexibility, tunable material properties and functionality of the microdevice allow the re-creation of characteristic biological niches found in native human tissue [4]. This aspect has direct significance for future medicine because it offers new insights into disease mechanisms as well as opportunities for advanced drug screening [5], and personalized and precision medicine [6].

This minireview looks at the progress made in organ-on-a-chip technology in the past two years, focusing on clinically-relevant medical or pharmaceutical use, including latest developments in physiologic organ-on-a-chip systems, as well as high-throughput screening, disease-modelling and personalized medicine.

\section{Human physiology on-a-chip: Status quo 2018}

Organ-on-a-chip technology reliably mimics the smallest functional unit of an organ with the physical, cellular and biochemical microenvironment accounting for the respective native tissue architecture for organs such as lung,[7] liver,[8] gut,[9,10] kidney,[11] artery,[12] 
heart,[13,14] placenta,[15,16] and others. Until recently, on-chip monolayer cultures were predominantly used on membranes to investigate the physiology, integrity and function of human cell barriers using mostly cancer cell lines [17]. However, since physiological responses cannot be guaranteed when using cancer cells in organ-on-a-chip systems, advanced organ-models nowadays are based on primary cells or induced pluripotent stem cells.

In the case of neurological microsystems, a variety of primary cell types and chip designs were employed to model simplified architecture and specific functions of the human brain. Under fluid shear, brain endothelial cells can be co-cultured with astrocytes on a porous hollow fiber resulting in a barrier with in vivo-like integrity and permeability [18]. By integrating more specialized and localized brain tissue models, such as the hippocampus, prefrontal cortex and amygdala, the permeability of potentially toxic agents can be tested using a multi-regional brain-on-a-chip device [19]. Despite this and other proof-of-principle studies, medical applications of such complex systems are still challenging [20,21]. For instance, one major challenge is the complexity of the three-dimensional structure of a nephron in pharmacological kidney research, which still presents an insurmountable hurdle in bioengineering despite recent developments in bioprinting techniques [22-25]. Although the complexity of the nephron cannot be recreated, drug clearance can nonetheless be modelled on a chip using a simpler microfluidic cell barrier system exhibiting basic organ function [11]. Another approach employed three-dimensional culture techniques based on scaffolding collagen I matrices and cellular self-organization to establish a kidney-on-a-chip system displaying clinically relevant biomarkers for toxicity for cis-platin and doxorubicin [26]. An alternative method of mimicking the kidney is based on using pluripotent stem cells to generate organoids containing nephron-like tubular structures [27]. In this case, cells were cultured in an air-liquid interface yielding robust tubulogenesis and nephron-like renal cells with podocyte-like progenitors from the glomeruli, as well as proximal tubular- and distal tubular-like cells. However, the presence of pre-maturity biomarkers indicated nonphysiological phenotypes.

An often overlooked aspect of every organ-on-a-chip system is the fact that every tissue and organ within the human body is subjected to biophysical forces, which are also key for 
development of proper physiological phenotypes [28]. Microfluidic devices containing integrated mechanical actuators have been developed with the ability to apply shear, strain, stretch and compression forces on cells to mimic mechanobiological cues [29,30]. The most straight forward strategy uses fluid flow to apply varying shear forces. For instance, fluid shear and channel curvature influence phenotype as well as transport function of renal proximal tubule models $[31,32]$. The first breakthrough organ-on-a-chip model that recreated organ biomechanics was a breathing lung-on-a-chip that used a lateral vacuum to exert strain on a thin flexible membrane and stimulate the lung epithelial cell barrier [7]. Following a period of two-weeks, increased epithelial surfactant production was induced by the continuous stretching motion, which resulted in improved barrier stability. A similar organ-on-a-chip principle was used to create a biomimetic human gut-on-a-chip mimicking the complex structure and peristaltic of native intestine tissue [33].

To overcome the technological challenge of parallelization of complex organ structures, different organ cultures were integrated in a single device to increase throughput [34]. Using pneumatic medium circulation, the pro-drug 5-fluorouracil and anti-cancer drugs capecitabine and tegafur were pumped between (a) cancer, (b) intestine and (c) connective tissue models to simultaneously screen for the efficacy and adverse effects of drug candidates. Another micro-physiological system with higher throughput capability was developed and comprised various vascularized micro tumor models [35]. Both anti-angiogenic and anti-tumor drug activities were successfully identified by blind-screening a small compound library using hydrostatic pumping. Additionally, a microfluidic hanging drop array was established for multi-parametric monitoring of multiple spheroidal models by integration of micropumps [36] and inline electrochemical biosensors based on amperometry [37] and electric impedance sensing [38]. Highest throughput is currently achieved using a fully automated cell culture platform engineered around Mimetas' OrganoPlate ${ }^{\circledR}$ system, which is optimized for longterm maintenance and monitoring of induced pluripotent stem cell differentiation into neuronal cultures using 96 individually addressable three-dimensional microfluidic cell cultures. This system was used to monitor the onset and progression of Parkinson's disease using patient-derived neuroepithelial stem cells that were differentiated into midbrain specific dopaminergic neurons over 24 days [39]. 


\section{Emulating human disease on-a-chip}

Disease modeling has been largely pioneered in animal experiments and conventional monolayer cell cultures. As mentioned above, three-dimensional in vitro cell cultures techniques potentially obviate animal models, because in situ pathology can be recreated using commercially available or patient derived cells bypassing significant intra-species differences between humans and animals [40]. An artificial bottom-up approach is frequently used to build organ-on-a-chip models with progressive complexity by exploitation of cellular differentiation, self-assembly or cell-to-cell interaction to recapitulate more features critical for disease etiology and progression [3]. For instance, organ-on-a-chip systems have been applied to investigate thrombosis and coagulation processes. A recently published pneumatic microsystem allows for quantitative spatiotemporal insights into endothelium wounding and clot formation under both physiologically and pathologic conditions [41]. Even though clinically problematic, eptifibatide showed no effect on in vivo bleeding time in pre-clinical animal and clinical human trials as well as on-chip observations. Another pneumaticcontrolled microdevice enabled clinical whole blood analysis while inflammation for different stenotic conditions during onset of atherosclerosis [42]. The system is capable of discriminating healthy and inflamed blood samples based on leukocyte adhesion at $80 \%$ stenosis, however, biochemical stimuli were higher than physiological levels. Also, threedimensional bioprinting was applied to a more biomimetic thrombosis-on-a-chip model consisting of printed gelatin methacryloyl hydrogel micro-vessels [43]. Continuous perfusion with tissue plasmin activator led to dissolution of clots. In addition, co-cultured fibroblasts that migrate and deposit collagen type I into the clot facilitated remodeling of fibrosis similar to in vivo. A two-compartment organ-on-a-chip was further established maintaining primary epithelial-vessel interface for 12 days to study thrombus formation in lung, which is a difficult process to investigate clinically [44]. Responses of this tissue-tissue interface to lipopolysaccharide exposure to the alveolar epithelium disrupted underlying endothelial cellcell junctions, and stimulated platelet aggregation and thrombus formation. No response was observable in the absence of the alveolar epithelium. This tissue level communication and response within the chip is in line with similar in vivo observations in a mouse vessel injury model $[45,46]$. Trauma of pulmonary vasculature during blood infusion poses another risk for 
lungs in a clinical setup. A micro-engineered disease-on-a-chip model culturing primary human lung endothelial cells demonstrated that red blood cell infusion leads to abnormal cytoskeletal rearrangement and release of intracellular molecules, that resembled the characteristics of acute vascular trauma of transfused lungs in vivo [47]. Another disease-ona-chip model covered with lined human intestinal epithelial cells and vascular endothelial cells was established to mimic acute $\gamma$-radiation syndrome, associated with gastrointestinal disturbances leading to intestinal villi change, disruption of barrier integrity, endothelial apoptosis, as well as mucosal barrier breakdown [48]. Not only did dimethyloxaloylglycine treatment significantly reduce irradiation related injuries and cellular abnormalities, an exposure of 8 Grey was comparable to structural and molecular changes found in irradiated intestinal tissue in vivo [49].

To elucidate cancer progression and metastasis, microfluidic platforms have been applied over the last two decades for cancer extravasation mechanisms on-chip for both, cellular and biochemical levels [4]. Cancer-on-a-chip system based on different organ tissues enable significant improvements to other in vitro disease model systems with more control over the cancer and tissue microenvironment and greater relevance to sophistication and heterogeneity of human cancer. For instance, a microfluidic platform with ossified tissues based on human osteoblasts was created to recapitulated important features of the myeloma microenvironment with bone marrow mononuclear cells and plasma collected from three multiple myeloma patients [50]. Bone marrow mononuclear cells from myeloma patients reduced tissue mineralization, which indicates that myeloma cells activated mechanisms compromising osteoblast activity. Even though these results look promising, clinical relevance is not clear to date because there is limited knowledge in vivo about the delicate balance between osteoblast and osteoclast activity leading to tumor-induced osteolysis in multiple myeloma [51].

\section{Organ-on-a-chip enabling personalized and precision medicine?}

Since the Nobel Prize in Medicine in 2012 was awarded for the discovery that mature cells can be reprogrammed to become pluripotent stem cells, patients-derived cells can be harvested and 
reprogrammed to become any tissue type found in the human body. Patient-tailored individualized remodeling of human health and disease based on organ-on-a-chip models enables personalized medicine based on generation of more reliable and individual compound screening outcomes, thus identification of the most effective individualized therapy.

For instance, separated on-chip co-cultures of myeloma and bone marrow cells as multiple myeloma model demonstrated that in vitro responses to bortezomib correlated well with clinical response of the patients only when the myeloma co-culture contained exclusively CD138-positive bone marrow cells [52]. A similar study combined patient-derived myeloma cells and stromal cells to analyze the effects of alkylating agent melphalan and the proteasome inhibitor bortezomib. Outcomes surprisingly revealed that co-culture of donor-matched primary myeloma with stromal cells increased myeloma cell resistance to melphalan. Interestingly, myeloma cells harvested at different tumor stages responded differently to anti-cancer drugs. Recently, a complex glomerular barrier model was bioengineered based on isolated glomerulus tissue spheroids from kidneys of adult rats, that histologically resemble native in vivo tissue, were captured and in turn cultured long-term as glomerular filtration barriers in multiple hydrogel pockets [53]. During hyperglycemic culture conditions the established filtration barriers mimicked diseased phenotypes in vivo for glomerulus endothelial cells and podocytes closely resembling reactive oxygen production and protein leakage of pathological responses seen in patients. A smoking human small airway-on-a-chip modelling chronic obstructive pulmonary disease was established for conventional as well as e-cigarette smoke [54]. Using this automated breathing and smoking microsystem, healthy or diseased patient bronchial epithelium was subjected to smoke showing striking similarities in gene alterations including aldo-keto reductase and cytochrome P450.

\section{Conclusions}

Chip-based in vitro organ models are ranked $6^{\text {th }}$ among the top ten emerging technologies by the World Economic Forum in 2016, thus highlighting the potential of organ-on-a-chips to improve lives and transform the health care system. In other words, organ-on-a-chip technology is expected to speed up pharmaceutical drug development efforts, improve translation of basic research to clinically relevant patient scenarios and provide personalized intervention strategies. Over the last two years, big pharma companies including Pfizer, Johnson/Johnson, Merck, Roche and Takeda 
Pharmaceutical have already engaged in academic research-cooperation to develop various organon-a-chip systems for future pharmaceutical drug discoveries. Although many organ-on-a-chip systems present elegant proof-of-concept studies, their translational relevance and predictive power remain to be demonstrated in clinical and pharmaceutical settings. This means that next generation of patient-derived organ-on-a-chip systems will need to reliably identify the most efficient and effective drug candidates from a large compound library to predict effective clinical outcomes. To reach this goal, organ-on-a-chip systems need to (a) employ primary cell types exhibiting (patho)physiological pheno- and genotypes, (b) provide an appropriate biological niche that promotes organ-relevant functions over extended periods of time and (c) allows for reproducible and reliable operation and analysis of a large number of organ models under physiological conditions. We anticipate that high-throughput and high-content of organ-on-chip models will be gradually introduced in the near future as outlined in Figure 1.

\section{Acknowledgements}

This work was supported by the European Union's Horizon 2020 Research and Innovation Programme [685817]; the Austrian Research Promotion Agency (FFG) [849791]; and EU Joint Programme - Neurodegenerative Disease Research (JPND) [3DPD; 735840]. 


\section{References}

1. Sticker D, Rothbauer M, Lechner S, Hehenberger MT, Ertl P: Multi-layered, membraneintegrated microfluidics based on replica molding of a thiol-ene epoxy thermoset for organ-on-a-chip applications. Lab on a Chip - Miniaturisation for Chemistry and Biology 2015, 15:4542-4554.

2. (*) Rothbauer M, Charwat V, Ertl P: Cell Microarrays for Biomedical Applications. Methods Mol Biol 2016, 1368:273-291.

3. Benam KH, Dauth S, Hassell B, Herland A, Jain A, Jang KJ, Karalis K, Kim HJ, MacQueen L, Mahmoodian R, et al.: Engineered in vitro disease models. Annu Rev Pathol 2015, 10:195-262.

4. (**) Rothbauer M, Zirath H, Ertl P: Recent advances in microfluidic technologies for cell-tocell interaction studies. Lab Chip 2017.

5. Rothbauer M, Wartmann D, Charwat V, Ertl P: Recent advances and future applications of microfluidic live-cell microarrays. Biotechnol Adv 2015, 33:948-961.

6. Wartmann D, Rothbauer M, Kuten O, Barresi C, Visus C, Felzmann T, Ertl P: Automated, Miniaturized, and Integrated Quality Control-on-Chip (QC-on-a-Chip) for CellBased Cancer Therapy Applications. Frontiers in Materials 2015, 2.

7. Huh D, Matthews BD, Mammoto A, Montoya-Zavala M, Yuan Hsin H, Ingber DE: Reconstituting organ-level lung functions on a chip. Science 2010, 328:1662-1668.

8. van Midwoud PA, Verpoorte E, Groothuis GMM: Microfluidic devices for in vitro studies on liver drug metabolism and toxicity. Integ Biol 2011, 3:509-521.

9. (*) Kim HJ, Lee J, Choi JH, Bahinski A, Ingber DE: Co-culture of Living Microbiome with Microengineered Human Intestinal Villi in a Gut-on-a-Chip Microfluidic Device. $J$ Vis Exp 2016.

10. Lee $\mathrm{J}$, Choi $\mathrm{JH}$, Kim HJ: Human gut-on-a-chip technology: will this revolutionize our understanding of IBD and future treatments? Expert Rev Gastroenterol Hepatol 2016, 10:883-885.

11. (*) Weber EJ, Chapron A, Chapron BD, Voellinger JL, Lidberg KA, Yeung CK, Wang Z, Yamaura Y, Hailey DW, Neumann T, et al.: Development of a microphysiological model of human kidney proximal tubule function. Kidney International 2016, 90:627-637.

12. Günther A, Yasotharan S, Vagaon A, Lochovsky C, Pinto S, Yang J, Lau C, VoigtlaenderBolz J, Bolz SS: A microfluidic platform for probing small artery structure and function. Lab Chip 2010 10:2341-2349.

13. Jastrzebska E, Tomecka E, Jesion I: Heart-on-a-chip based on stem cell biology. Biosens Bioelectron 2016, 75:67-81.

14. Marsano A, Conficconi C, Lemme M, Occhetta P, Gaudiello E, Votta E, Cerino G, Redaelli A, Rasponi M: Beating heart on a chip: a novel microfluidic platform to generate functional 3D cardiac microtissues. Lab Chip 2016, 16:599-610.

15. Lee JS, Romero R, Han YM, Kim HC, Kim CJ, Hong JS, Huh D: Placenta-on-a-chip: a novel platform to study the biology of the human placenta. $J$ Matern Fetal Neonatal Med 2016, 29:1046-1054.

16. Sticker D, Rothbauer M, Lechner S, Hehenberger MT, Ertl P: Multi-layered, membraneintegrated microfluidics based on replica molding of a thiol-ene epoxy thermoset for organ-on-a-chip applications. Lab Chip 2015, 15:4542-4554. 
17. Odijk M, van der Meer AD, Levner D, Kim HJ, van der Helm MW, Segerink LI, Frimat J-P, Hamilton GA, Ingber DE, van den Berg A: Measuring direct current trans-epithelial electrical resistance in organ-on-a-chip microsystems. Lab on a Chip 2015, 15:745-752.

18. Cucullo L, Hossain M, Rapp E, Manders T, Marchi N, Janigro D: Development of a humanized in vitro blood-brain barrier model to screen for brain penetration of antiepileptic drugs. Epilepsia 2007, 48:505-516.

19. Dauth S, Maoz BM, Sheehy SP, Hemphill MA, Murty T, Macedonia MK, Greer AM, Budnik B, Parker KK: Neurons derived from different brain regions are inherently different in vitro: a novel multiregional brain-on-a-chip. J Neurophysiol 2017, 117:1320-1341.

20. Kilic O, Pamies D, Lavell E, Schiapparelli P, Feng Y, Hartung T, Bal-Price A, Hogberg HT, Quinones-Hinojosa A, Guerrero-Cazares H, et al.: Brain-on-a-chip model enables analysis of human neuronal differentiation and chemotaxis. Lab Chip 2016, 16:41524162.

21. (**) Adriani G, Ma D, Pavesi A, Kamm RD, Goh EL: A 3D neurovascular microfluidic model consisting of neurons, astrocytes and cerebral endothelial cells as a blood-brain barrier. Lab Chip 2017, 17:448-459.

22. Peloso A, Katari R, Murphy SV, Zambon JP, DeFrancesco A, Farney AC, Rogers J, Stratta RJ, Manzia TM, Orlando G: Prospect for kidney bioengineering: shortcomings of the status quo. Expert Opin Biol Ther 2015, 15:547-558.

23. Gao G, Huang Y, Schilling AF, Hubbell K, Cui X: Organ Bioprinting: Are We There Yet? Adv Healthc Mater 2018, 7.

24. Xia Z, Jin S, Ye K: Tissue and Organ 3D Bioprinting. SLAS Technol 2018:2472630318760515.

25. Ovsianikov A, Khademhosseini A, Mironov V: The Synergy of Scaffold-Based and Scaffold-Free Tissue Engineering Strategies. Trends Biotechnol 2018, 36:348-357.

26. Adler M, Ramm S, Hafner M, Muhlich JL, Gottwald EM, Weber E, Jaklic A, Ajay AK, Svoboda D, Auerbach S, et al.: A Quantitative Approach to Screen for Nephrotoxic Compounds In Vitro. J Am Soc Nephrol 2016, 27:1015-1028.

27. Taguchi A, Kaku Y, Ohmori T, Sharmin S, Ogawa M, Sasaki H, Nishinakamura R: Redefining the in vivo origin of metanephric nephron progenitors enables generation of complex kidney structures from pluripotent stem cells. Cell Stem Cell 2014, 14:53-67.

28. Wall M, Butler D, El Haj A, Bodle JC, Loboa EG, Banes AJ: Key developments that impacted the field of mechanobiology and mechanotransduction. J Orthop Res 2018, 36:605-619.

29. Ertl P, Sticker D, Charwat V, Kasper C, Lepperdinger G: Lab-on-a-chip technologies for stem cell analysis. Trends Biotechnol 2014, 32:245-253.

30. Sticker D, Lechner S, Jungreuthmayer C, Zanghellini J, Ertl P: Microfluidic Migration and Wound Healing Assay Based on Mechanically Induced Injuries of Defined and Highly Reproducible Areas. Anal Chem 2017, 89:2326-2333.

31. Shen C, Meng Q, Zhang G: Increased curvature of hollow fiber membranes could upregulate differential functions of renal tubular cell layers. Biotechnol Bioeng 2013, 110:2173-2183.

32. Raghavan V, Rbaibi Y, Pastor-Soler NM, Carattino MD, Weisz OA: Shear stress-dependent regulation of apical endocytosis in renal proximal tubule cells mediated by primary cilia. Proc Natl Acad Sci U S A 2014, 111:8506-8511. 
33. (**) Kim HJ, Huh D, Hamilton G, Ingber DE: Human gut-on-a-chip inhabited by microbial flora that experiences intestinal peristalsis-like motions and flow. Lab Chip 2012, 12:2165-2174.

34. (*) Satoh T, Sugiura S, Shin K, Onuki-Nagasaki R, Ishida S, Kikuchi K, Kakiki M, Kanamori T: A multi-throughput multi-organ-on-a-chip system on a plate formatted pneumatic pressure-driven medium circulation platform. Lab on a Chip 2018, 18:115-125.

35. (*) Phan DTT, Wang X, Craver BM, Sobrino A, Zhao D, Chen JC, Lee LYN, George SC, Lee $\mathrm{AP}$, Hughes CCW: A vascularized and perfused organ-on-a-chip platform for largescale drug screening applications. Lab Chip 2017, 17:511-520.

36. Yazdi SR, Shadmani A, Burgel SC, Misun PM, Hierlemann A, Frey O: Adding the 'heart' to hanging drop networks for microphysiological multi-tissue experiments. Lab on a Chip 2015, 15:4138-4147.

37. Misun PM, Rothe J, Schmid YRF, Hierlemann A, Frey O: Multi-analyte biosensor interface for real-time monitoring of 3D microtissue spheroids in hanging-drop networks. 2016, 2:16022.

38. Schmid YRF, Bürgel SC, Misun PM, Hierlemann A, Frey O: Electrical Impedance Spectroscopy for Microtissue Spheroid Analysis in Hanging-Drop Networks. ACS Sensors 2016, 1:1028-1035.

39. Kane KIW, Lucumi Moreno E, Hachi S, Walter M, Jarazo J, Hankemeier T, Vulto P, Schwamborn J, Thoma M, Fleming RMT: Automated microfluidic cell culture of stem cell derived dopaminergic neurons in Parkinsons disease. bioRxiv 2017, doi: doi:10.1101/209957.

40. Seok J, Warren HS, Cuenca AG, Mindrinos MN, Baker HV, Xu W, Richards DR, McDonaldSmith GP, Gao H, Hennessy L, et al.: Genomic responses in mouse models poorly mimic human inflammatory diseases. Proc Natl Acad Sci U S A 2013, 110:3507-3512.

41. (**) Sakurai Y, Hardy ET, Ahn B, Tran R, Fay ME, Ciciliano JC, Mannino RG, Myers DR, Qiu Y, Carden MA, et al.: A microengineered vascularized bleeding model that integrates the principal components of hemostasis. Nat Commun 2018, 9:509.

42. (*) Venugopal Menon N, Tay HM, Pang KT, Dalan R, Wong SC, Wang X, Li KHH, Hou HW: A tunable microfluidic 3D stenosis model to study leukocyte-endothelial interactions in atherosclerosis. APL Bioengineering 2018, 2:016103.

43. Zhang YS, Davoudi F, Walch P, Manbachi A, Luo X, Dell'Erba V, Miri AK, Albadawi H, Arneri A, Li X, et al.: Bioprinted thrombosis-on-a-chip. Lab Chip 2016, 16:4097-4105.

44. Jain A, Barrile R, van der Meer AD, Mammoto A, Mammoto T, De Ceunynck K, Aisiku O, Otieno MA, Louden CS, Hamilton GA, et al.: Primary Human Lung Alveolus-on-a-chip Model of Intravascular Thrombosis for Assessment of Therapeutics. Clin Pharmacol Ther 2018, 103:332-340.

45. Cooley BC: In vivo fluorescence imaging of large-vessel thrombosis in mice. Arterioscler Thromb Vasc Biol 2011, 31:1351-1356.

46. Abrams ST, Zhang N, Manson J, Liu T, Dart C, Baluwa F, Wang SS, Brohi K, Kipar A, Yu W, et al.: Circulating Histones Are Mediators of Trauma-associated Lung Injury. American Journal of Respiratory and Critical Care Medicine 2013, 187:160-169.

47. Seo J, Conegliano D, Farrell M, Cho M, Ding X, Seykora T, Qing D, Mangalmurti NS, Huh D: A microengineered model of RBC transfusion-induced pulmonary vascular injury. Sci Rep 2017, 7:3413. 
48. Jalili-Firoozinezhad S, Prantil-Baun R, Jiang A, Potla R, Mammoto T, Weaver JC, Ferrante TC, Kim HJ, Cabral JMS, Levy O, et al.: Modeling radiation injury-induced cell death and countermeasure drug responses in a human Gut-on-a-Chip. Cell Death Dis 2018, 9:223.

49. Jang H, Park S, Lee J, Myung JK, Jang WS, Lee SJ, Myung H, Lee C, Kim H, Lee SS, et al.: Rebamipide alleviates radiation-induced colitis through improvement of goblet cell differentiation in mice. J Gastroenterol Hepatol 2018, 33:878-886.

50. Zhang W, Lee WY, Siegel DS, Tolias P, Zilberberg J: Patient-specific 3D microfluidic tissue model for multiple myeloma. Tissue Eng Part C Methods 2014, 20:663-670.

51. Papadopoulou EC, Batzios SP, Dimitriadou M, Perifanis V, Garipidou V: Multiple myeloma and bone disease: pathogenesis and current therapeutic approaches. Hippokratia 2010, 14:76-81.

52. Pak C, Callander NS, Young EW, Titz B, Kim K, Saha S, Chng K, Asimakopoulos F, Beebe DJ, Miyamoto S: MicroC(3): an ex vivo microfluidic cis-coculture assay to test chemosensitivity and resistance of patient multiple myeloma cells. Integr Biol (Camb) 2015, 7:643-654.

53. (*) Wang L, Tao T, Su W, Yu H, Yu Y, Qin J: A disease model of diabetic nephropathy in a glomerulus-on-a-chip microdevice. Lab Chip 2017, 17:1749-1760.

54. Benam KH, Novak R, Nawroth J, Hirano-Kobayashi M, Ferrante TC, Choe Y, Prantil-Baun R, Weaver JC, Bahinski A, Parker KK, et al.: Matched-Comparative Modeling of Normal and Diseased Human Airway Responses Using a Microengineered Breathing Lung Chip. Cell Syst 2016, 3:456-466 e454. 
Figure 1. Requirements for next generation organ- and disease-on-a-chip models for clinically relevant drug screening applications and personalized medicine. 


\section{Next generation of Organs-on-a-chip}
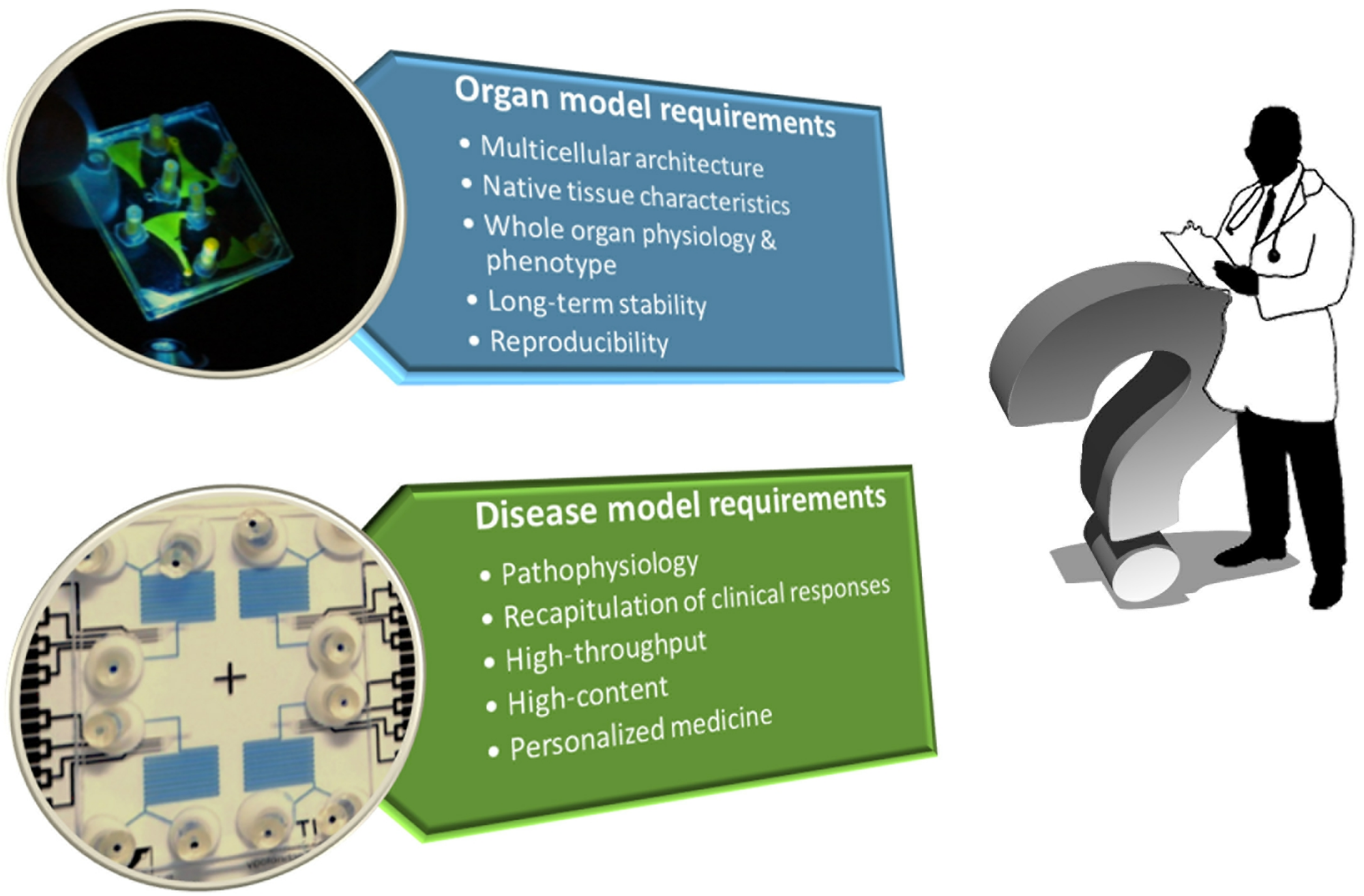